This document is the accepted manuscript version of the

following article:

Masinel1i, G., Shevchik, S., Pandiyan, V., Quang-Le, T., \&

wasmer, K. (2021). Artificial intelligence for monitoring and control of metal additive manufacturing. In M. Meboldt \& C. Klahn (Eds.), Industrializing additive manufacturing. Proceedings of AMPA2020 (pp. 205-220).

https://doi .org/10.1007/978-3-030-54334-1_15

\title{
Artificial Intelligence for Monitoring and Control of Metal Additive Manufacturing
}

\author{
G. Masinelli, S. Shevchik, V. Pandiyan, T. Quang-Le, and K. Wasmer
}

Laboratory of Advanced Materials Processing, Swiss Federal Laboratories for Materials Science and Technology (Empa), 3602 Thun, Switzerland,

kilian.wasmer@empa.ch,

WWW home page: https://www.empa.ch/web/s204/dynamical-processes

\begin{abstract}
Quality monitoring in Additive Manufacturing (AM) is currently mostly based on temperature measurements of the process zone or on layer/piecewise high-resolution surface imaging. To this aim, various sensors, such as pyrometers, photodiodes, and matrix CCD detectors, have been tested. These standard temperature measurements, however, do not provide a comprehensive description of the process dynamics, as they are just limited to surface observations. Furthermore, they are often used for post-factum inspection, i.e., after the piece is partially or even completely produced. No robust and low-cost methods are so far known to monitor the quality of laser processes in real-time. To close this gap, we propose an innovative approach for online quality monitoring of additive manufacturing employing acoustic emissions (AE). In fact, $\mathrm{AE}$ signals can provide in-depth information about the process, e.g., melting, resolidification, delamination, and cracking of the workpiece. Moreover, the sintering or melting of the metal powder has several unique acoustic signatures that can be detected and interpreted in terms of quality. In our approach, the correlation of the acoustic signals to the quality of the produced pieces is made by Artificial Intelligence (AI) methods. Specifically, AI in the form of Machine Learning is used to perform a data-driven extraction and recognition of the unique acoustic signatures from different sintering or melting events. In this contribution, we present a summary of our results in the fields of selective laser melting and laser welding, which have similar underlying mechanisms. At first, we discuss how, by using AE, we can classify different types of defects and porosity content in both processes. Afterward, with the aid of high-speed X-ray imaging, we demonstrate the real-time performance of our approach in the classification of transient events/regimes that are critical for the final quality - in particular conduction, stable keyhole, unstable keyhole, pore formation, and blowout. Finally, we present the future possibilities in terms of control of AM processes based on AI.
\end{abstract}

Keywords: Additive manufacturing, process monitoring, process control, acoustic emission, Artificial Intelligence 


\section{Introduction}

Additive Manufacturing (AM) is a technology developed to manufacture threedimensional components based on computer models with great flexibility on the choice of materials, including metals, ceramics, and polymers in different forms, such as liquids, powders, or wires. AM offers several advantages compared to traditional manufacturing methods [1, including very few geometric shape limitations [2, innovative multi-material manufacturing, and low running costs. These characteristics meet the needs of modern production, to the point that AM is considered to be the new industrial revolution [1], drawing the attention of a growing number of industries, such as automotive [3], medical [4], aerospace [5], jewelry [6], and food [7].

In this article, we focus on Selective Laser Melting (SLM) 8 - an AM subbranch that allows building metallic components from powders. Similarly to other AM technologies, the underlying physical phenomena that enable the functioning of SLM, are highly complex. Indeed, they involve the interaction between the intense laser beam with multiple material phases (solid, liquid, and vapor), as well as very fast heating and cooling cycles of the base material (from $10^{3}$ to $\left.10^{7} \mathrm{~K} / \mathrm{s}\right) 9$.

Therefore, despite many developments of this technology, SLM still suffers from imperfect process repeatability $10-12$, limiting its applications in mass production, in particular in the ones with high-quality standards. Indeed, many processing parameters - e.g., laser energy, scanning speed, hatch distance, and powder layer thickness - must be carefully chosen according to the specific powder used [2, 13, 14]. An improper choice of the parameters can lead to an accumulation of residual stress inside the workpiece or to the formation of defects, such as significant porosity, cracks, or lack of fusion [11], resulting in weakened mechanical properties 15 .

Currently, the industrial standard for the quality monitoring of the parts produced by AM in terms of porosity or cracking is X-ray tomography [16]. Being a costly and time-consuming process, this type of analysis is generally performed post-mortem - after the part has already been produced. In the literature, there are also some attempts to design near real-time quality monitoring systems 11, 17. The two main adopted approaches are: i) image analysis of the surface of each layer of the workpiece [18, and ii) temperature measurements of the melting zone [11]. The main drawbacks of both techniques derive from their limitation to surface measurements, with limited access to in-depth information. Furthermore, their temporal resolution is not ideal for the analysis of the transient events during the process, which have been reported to take place at time scales down to hundreds of microseconds [19]. For these reasons, the accuracy of those commercial approaches is debatable [11].

In this context, there is a clear need for a reliable, cost-effective, and efficient detection system that can provide subsurface information about the process. One solution can be found in Acoustic Emission (AE) sensing technology. Indeed, the use of AE signals to trace the onset or propagation of cracks/defects in nontransparent materials is well-known for its efficiency, high sensitivity, and non- 
destructive nature [20,21]. Moreover, it can be easily implemented in industrial environments for online monitoring due to low-cost hardware. The main obstacles that prevent the wide-spread use of AE technology for AM monitoring derive from weak useful signals and loud background noise.

To this extent, we proposed a new methodology where AE is combined with Machine Learning (ML). Indeed, highly sensitive AE sensors combined with ML enable the detection of meaningful information in AM signals even in a very loud environment. This approach has already been successfully applied to several applications with a high level of background noise, including tribology [22, and fracture mechanics 23 . It has to be noted that - even though the selected examples used a highly sensitive fiber-based detector - the proposed approach is very flexible in terms of sensing devices, from piezo-based contact sensors to airborne microphones.

This contribution summarizes the work done so far by our research group in the field of in situ AM and Laser Welding (LW) monitoring, combining AE and ML. We start with the retrospective of the preliminary feasibility study and then highlight the recent developments in correlating $\mathrm{AE}$ with the hidden physical mechanisms responsible for the defects formation.

Firstly, we begin with an overview of the work of Shevchik et al. 24, which is a preliminary study to investigate the feasibility of discerning, from AE signals, between three different final build qualities in terms of porosity. The different quality grades were intentionally provoked by using three sets of process parameters. Secondly, the application of our approach for the monitoring of different LW regimes is presented 25]. Even though this work refers to a different application, the same physical phenomena are involved in melting and subsequent solidification of the metallic material. Thirdly, we move to a more recent work, 26, where the AE based monitoring system was pushed a step further, aiming at identifying the momentary events leading to the formation of defects, such as porosity and blowout. In this case, the system was guided, during its training, by high-speed X-ray radiography. In particular, it was shown that our methodology could distinguish between stable and unstable keyhole regimes with high confidence levels. Finally, we conclude with a discussion on how the precedent results can be used for an AI-based closed-loop system for defect-free laser processes.

\section{Detection tool for Additive Manufacturing}

The work presented hereafter is focused on SLM, but the same concepts can also be applied to many other AM techniques. We start the survey from the work of Shevchik et al. 24 that investigated the feasibility of in situ monitoring of SLM processes combining AE and ML. 


\subsection{Experimental setup}

To validate the robustness of the method, several experiments were carried out using a commercially available machine Concept M2 (Concept Laser GmbH, Germany), equipped with a fiber laser that operates continuously at a wavelength of $1071 \mathrm{~nm}$. The diameter of the focused laser spot was $90 \mu \mathrm{m}$, and the beam quality factor was $M^{2}=1.02$. The powder material was CL20ES stainless steel $(1.4404$ / 316L) with a particle size distribution ranging from 10 to $45 \mu \mathrm{m}$. The produced experimental sample had a cuboid shape with dimensions of 10 $\times 10 \times 20 \mathrm{~mm}^{3}$.

In this work, the quality aspect of interest was the concentration of pores in the workpiece. Their presence is one of the most critical defects as they may not be noticed during a visual inspection, and they harm the mechanical integrity of the sample. Different porosity levels were induced by using diverse laser scanning velocities during the manufacturing of the various layers of the workpiece, all the while fixing other process parameters (laser power $P=125 \mathrm{~W}$, hatch distance $h=0.105 \mathrm{~mm}$, powder layer thickness $t=0.03 \mathrm{~mm}$ ). Fig. 1 shows a typical optical image taken at the cross-section of the workpiece.
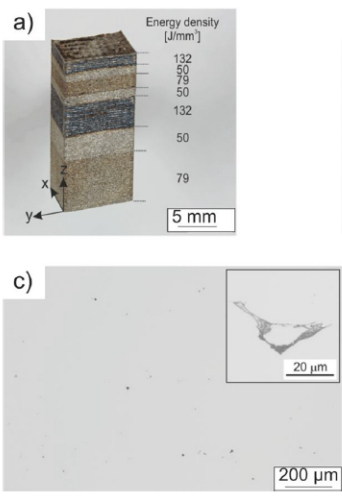
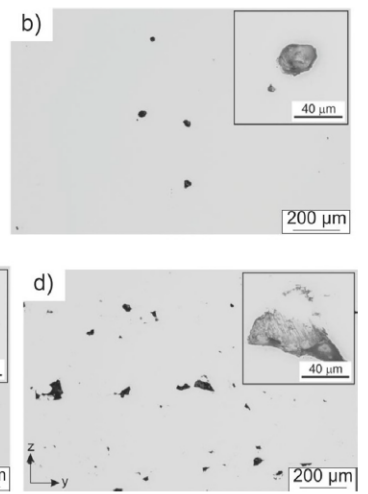

Fig. 1. (a) Test workpiece produced with three porosity concentrations. The regions with different qualities can be distinguished by their unique colors. Typical light microscope cross-section images of the regions produced with a laser scanning speed of (b) $300 \mathrm{~mm} / \mathrm{s}$ (medium quality), (c) $500 \mathrm{~mm} / \mathrm{s}$ (high quality), and (d) $800 \mathrm{~mm} / \mathrm{s}$ (poor quality). Reprinted by permission from Elsevier License: Elsevier 24].

Poor quality refers to the process parameters leading to the highest pore concentration $(1.42 \%)$ and is obtained with the highest scanning speed $(800 \mathrm{~mm} / \mathrm{s})$. In this case, the cause of the porosity is often referred to as lack of fusion, and occured due to insufficient energy to sinter all the particles within the laser beam. As far as the medium quality is concerned, the porosity level was $0.3 \%$, and the pores were caused by the overheating of the material due to the lower laser scanning speed and, consequently, higher dose of deposited energy, resulting in 
the formation of deep keyhole channels. Finally, good samples were obtained using an average scanning speed between the two aforementioned ones, and the achieved porosity level was $0.07 \%$. In this case, the speed was selected to provide, on the one hand, sufficient energy to sinter all the particles within the laser beam, and on the other hand, to prevent the material from overheating. More details on the process parameters and their effect on the working piece can be found in Shevchik et al. 24] and in Fig. 1.

The AE signals during the entire SLM process were recorded with a Fiber Bragg Grating (FBG). The latter was placed inside the printing chamber, at a distance of $20 \mathrm{~cm}$ from the process zone. Detailed information about the sensing principle of the FBG sensor can be found in Shevchik et al. 24.

\subsection{Correlation of AE with AM quality}

The collected AE signals were divided into three categories according to the quality of the obtained pieces. The obtained dataset was stochastically split into two sets, one for training and one for testing. Each category of the dataset consisted of 300 signals of $160 \mathrm{~ms}$, and there was no shared data between the two datasets. This approach simulated a real-life condition in which the trained system has to operate with unseen input data.

\subsubsection{Feature extraction}

The mathematical transform known as Wavelet Package Transform (WPT) was used to extract meaningful information from the signals. In particular, the WPT allows calculating the spectrograms of each signal, consisting of the relative energies of different narrow frequency bands 27, 28. An example of a raw $\mathrm{AE}$ signal and its spectrogram computed by means of WPT can be found in Fig. 2 .
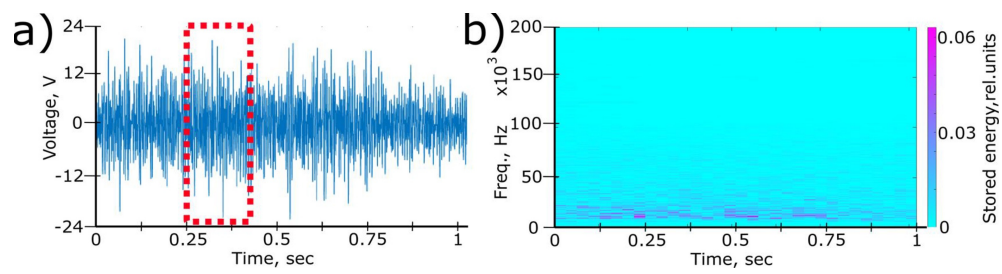

Fig. 2. Example of AE signal recorded when using the optimal process parameters (a) and the corresponding spectrogram made up of the signal's relative energies in several narrow frequency bands localized in time-frequency domain (b). The red marker in (a) shows the window of $160 \mathrm{~ms}$ used to partition the signals. Reprinted by permission from Elsevier License: Elsevier 24].

The signal representation provided by the WPT has several significant advantages. First, it reduces the amount of data and ambient noise by allowing the selection of specific frequency bands. In addition, it enables the interpretation of 
the AE signals as an evolution over time of the chosen frequency bands. Finally, it adapts the signals so that they can be used as inputs to existing Convolutional Neural Network (CNN) implementations. In fact, most existing CNNs are developed for image processing, where the 2D spatial domain of a picture is analyzed. Since the wavelet spectrogram domain is two-dimensional as well (time and frequency), the existing CNNs can be directly applied to them. More details on this technique can be found in Shevchik et al. 24].

\subsection{Spectral Convolutional Neural Networks (SCNN)}

The main disadvantage of using raw AE signals is their inherent complexity that originates from the complicated nature of the underlying mechanisms. Practically, this means that the differences between signals from different categories are not evident, making the classification task harder.

A conventional dimensionality reduction technique (Principal Component Analysis, PCA, 29]) makes it evident that data from different categories is very entangled, and no discrete clusters (data groupings) can be found (see Fig. 3). For these reasons, simple, but effective ML techniques such as SVM cannot be used.

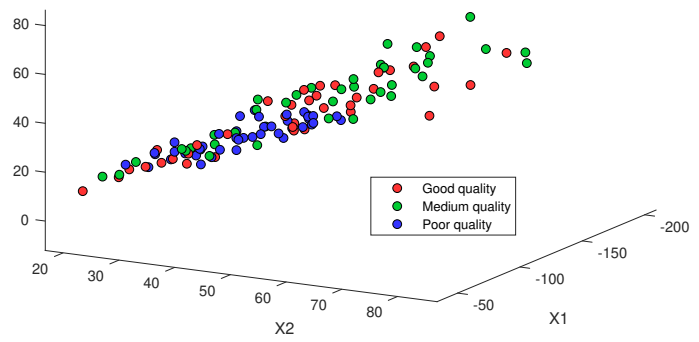

Fig. 3. Projection of the acoustic features into a 3D feature space using Principal Component Analysis (PCA). The visualized dataset includes thirty data points from each quality category. Reprinted by permission from Elsevier License: Elsevier [24].

Deep CNNs are well-known for being able to tackle this problem by successive projections of the data into higher dimensionality spaces 30 . The main disadvantage is that their training is very demanding in terms of both computational resources and amount of required data. To overcome these limits, a Spectral Convolutional Neural Network (SCNN) has been adopted. SCNN inherits all the advantages of traditional convolutional neural networks, with the additional benefit of being able to process data with more complex structures (or geometries) in a lightweight fashion in terms of computational effort. These benefits 
are obtained by using irregular convolutional operations as a feature extraction tool. In particular, the irregularity of the input data is captured using graphs. This external tool guides the network during the training procedure to optimize its structure. As a result, it gives the possibility to process highly irregular data in cases where the application of linear classifiers is not effective. More details on the network architecture can be found in Shevchik et al. 24.

\subsubsection{Results and limitations}

As described in Shevchik et al. 24], the overall accuracies of the SCNN application towards porosity detection ranged from $83 \%$ to $89 \%$. These results showed the potential of the proposed approach, especially considering that this was only the first feasibility study. In other words, we can conclude that the AE signals recorded by an FBG and their processing with the SCNN have the potential to be a solution for in situ quality monitoring of AM processes.

It has to be noted that the classification accuracy was high, but it can be furtherly improved. Indeed, medium quality samples are occasionally misclassified with the other categories. One of the aspects that limits the potential of the developed technique is the length of the acoustic signal acquisition window. In the presented study, it was observed that the optimal time span was $160 \mathrm{~ms}$. However, the window length is a trade-off between stability and spatial resolution. Indeed, reducing the window span increases the temporal resolution of the detection of the momentary events, but, unfortunately, so does the sensitivity to noises. To overcome those limitations, in Shevchik et al. [31, the structure of the developed SCNN is further developed to be able to process data from the combination of two scanning windows with different time spans.

\subsection{Double scanning strategy}

As previously mentioned, the choice of the time span of the scanning window is crucial. In fact, the AM process incorporates multiple events with different time scales, and a window of a fixed size can hardly capture all the transients involved. For this reason, in Shevchik et al. [31], the collected AE signals were scanned with two running windows that were characterized by different time lengths, indicated as short and long running window (SRW and LRW, respectively). By using two running windows at the same time, an analysis - which is stable to the presence of noise - is carried out at different time scales. This feature is particularly useful in the application under examination. Therefore, the CNN structure has been adapted to process the spectrograms calculated from both SRW and LRW simultaneously. Specifically, the wavelet spectrograms for SRW and LRW were constructed separately and were used as inputs for the SCNN classifier. Finally, an exhaustive search has been performed to identify the optimal windows sizes and resulted in timespans of $80 \mathrm{~ms}$ and $160 \mathrm{~ms}$ for SRW and LRW, respectively. An example of the two optimal running windows and a typical AE signal can be found in Fig. 4 . 


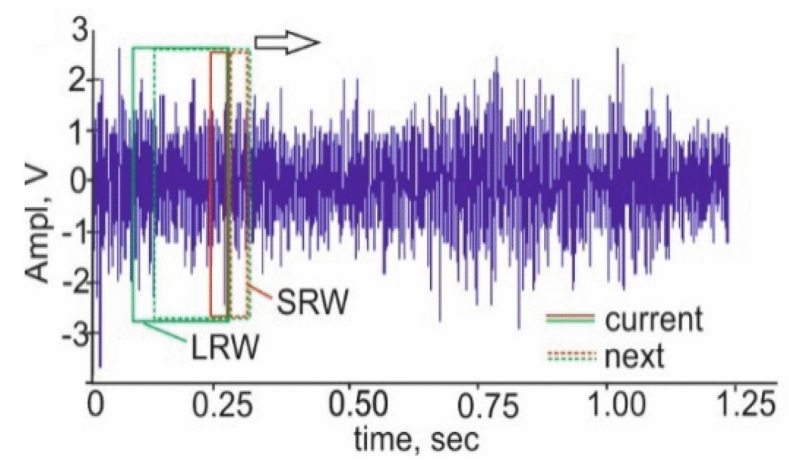

Fig. 4. Visualization of a typical raw AE signal and the optimal windows sizes of $80 \mathrm{~ms}$ and $160 \mathrm{~ms}$ for SRW and LRW. Reprinted by permission from IEEE License: IEEE [31].

\subsubsection{Results and limitations}

The classification accuracies obtained by this method range from $78 \%$ to $91 \%$. These results demonstrate that it is possible to combine highly sensitive $\mathrm{AE}$ sensors with an SCNN to classify the sample qualities in terms of porosity concentration. It has to be noted that the classification was carried out without using imaging techniques or altering the setup and with minimum manual interventions. Besides, the results have shown that the continuous SLM process can be divided into a finite number of momentary events with unique acoustic signatures. These signatures can be analyzed by the developed Machine Learning framework and interpreted in terms of quality. The developed technique is also able to detect porosity within the material, which cannot be achieved with existing image-based methods [11.

Given the similarities of SLM to laser welding of metal workpieces, a similar approach has been used for the detection of the different welding regimes during laser welding.

\section{$3 \quad$ Laser welding quality monitoring}

As described in Section 2.2 the developed ML technique allowed discerning between different operating regimes responsible for different qualities during the AM process. In the successive work [25], the authors aimed at exploiting the previously reported approach for a more challenging task, namely, to apply the monitoring system to LW to identify the relationships between the different welding regimes and the AE signals. The usage of the same methodology to this different application comes as no surprise, as LW is an industrial process with many similarities to SLM, involving the same physical mechanism for metal melting and successive solidification. In particular, in Shevchik et al. 25], the investigations were carried out using laser welding of titanium workpieces.

In this specific contribution, the signals collected during laser welding were assigned to four categories: (i) no illumination, (ii) conduction welding, (iii) keyhole 
without porosity, and (iv) keyhole with porosity. The first category represents the absence of laser radiation. The second one is characterized by a low laser intensity leading to the so-called conduction welding regime (shallow). The third and fourth categories refer to the keyhole (deep) welding regimes with and without the presence of pores in the workpiece.

The distinction between the different welding regimes is crucial because, in real applications, the transition between them can be provoked by local changes in the material properties or in process parameters. To be specific, the capability to distinguish between keyhole with and without porosity is of particular interest for industrial applications. Indeed, the keyhole welding regime, despite its advantages 32,33], is prone to defects. In particular, the high instability of the keyhole channel in deep welding can lead to porosity in the workpiece 34,35. In this work, the ground truth for the said categories was estimated a posteriori by the visual inspection of the cross-sections of the welding joints.

On par with the previous works, the WPT was used to compute the relative energies of the selected narrow frequency bands to form the spectrograms of each signal. In contrast, the ML classifier that processes the spectrograms was modified to boost the computational speed to enable the online monitoring of the process.

\subsection{Graph SVM with adaptive multi-kernel}

As already discussed in paragraph 2.3, the described classification task is challenging because of the similarities between data of different categories. Even though the problem has been solved by using an SCNN, its relatively high number of parameters and the computational resources needed for building a graph for every new input sample to be classified, make it not suitable for real-time applications.

For this reason, an original multi-kernel approach has been proposed in Shevchik et al. 25] to relax the computational constraints while keeping a high classification accuracy.

Specifically, in Shevchik et al. 25], the classification of the collected AE signals was carried out by a modification of the Laplacian Support Vector Machine (LapSVM) 36]. The attractiveness of LapSVM relies on resolving sophisticated features configurations by searching for the optimal decision cut in the feature space to differentiate between the various categories [37]. Nevertheless, on the other side, its main limitations derive from the general usage of a single Gaussian kernel. Indeed, even though it can adapt to complex geometries, it fails to recognize separated categories if the corresponding features are not well clustered. For these reasons, LapSVM was improved by involving several adaptive Gaussian kernels.

As shown in Shevchik et al. [25], this modification enhanced the algorithm abilities in separating features from different categories. A depictive example on how the multi-kernel LapSVM acts on the data can be found in Fig. 5. 
It is noteworthy to notice that the developed ML technique inherits from the conventional LapSVM a very high computational efficiency. Indeed, the classification of a new input data is reduced to just a matrix multiplication, allowing the monitoring system to work in real-time. To be specific, the computational time needed per measurement was as fast as $70 \mathrm{~ms}$.

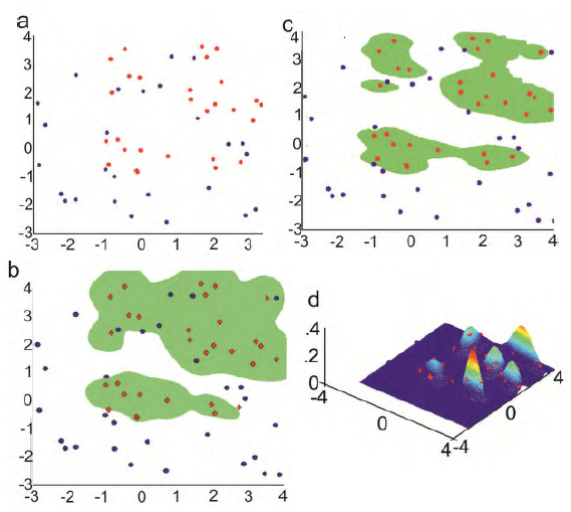

Fig. 5. Algorithm performance with synthetic data made up of two categories whose features do not form distinct groups: (a) original feature space, where the two colors represent the two categories; (b) decision boundaries defined by LapSVM with a single kernel; as can be seen, there are plenty of misclassifications, as several blue points fall inside the green region; (c) decision boundaries defined by LapSVM with multi Gaussian kernels; (d) 3D visualization of the learned Gaussian kernels after multikernel LapSVM training. Reprinted by permission from IEEE License: IEEE 25].

\subsubsection{Results and limitations}

As reported in Shevchik et al. [25, the classification accuracies of the real-time monitoring systems are very high, ranging from $87 \%$ to $99 \%$. However, the categories that were misclassified the most are keyhole with porosity and keyhole without porosity. These results indicate that there are overlapping of the $\mathrm{AE}$ features for those categories, which can be improved by optimizing the algorithms. On the other hand, the false classifications can also be due to errors in the identification of the ground truth. Indeed, it is almost impossible to detect all the porosities by optical inspection of the cross-sections, due to the small size of the pores. Moreover, the post mortem analysis cannot identify the pores that have been generated during the process and which have disappeared afterward when reaching the liquid/air interface [19,38].

In support of these assertions, it can be noted in Table 1 that almost $11 \%$ of the input data labeled by visual inspection as keyhole without porosity is classified as keyhole with porosity, while just $8 \%$ of the input data labeled as keyhole with porosity is mistaken for keyhole without porosity. 


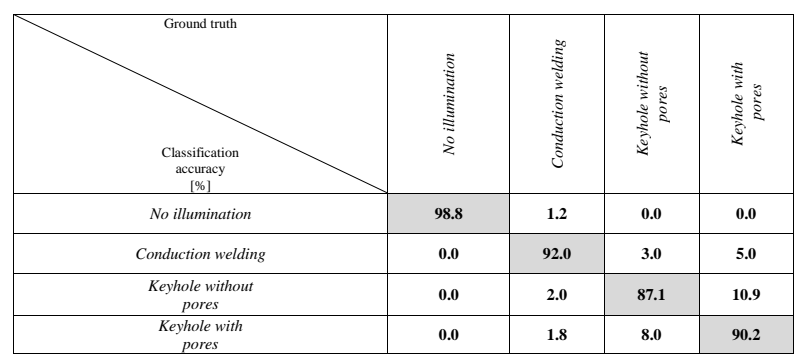

Table 1. Percentage classification accuracies per category versus ground truth. Adapted from Shevchik et al. 25].

\subsection{Quality monitoring with high-speed X-ray radiographic guidance}

It is noteworthy that all the research work done so far has been focused on a statistical approach to the analysis of the AE signals with the primary goal of monitoring. Therefore, the nature and the physical explanation of the $\mathrm{AE}$ contents were not investigated. In particular, the developed method allowed to stop a process when a non-acceptable defect is detected. However, if this happens, there are no other solutions than to discard the workpiece, despite the waste of materials and machining time. To overcome this issue, a fully automatic control loop is required. Nevertheless, to achieve this goal, we need to understand the physics of defect creation and to determine whether it is possible to classify the transients leading to artifacts such as the transition between stable and unstable keyhole.

Given the importance and relevance of the matter, the successive contributions of our research group were focused in this direction. In particular, in Shevchik et al. [26, the AE signals were analyzed employing ML techniques to identify the relationships between the momentary events that have an impact on the quality of the weld, and the AE signals. To provide the ML algorithms with precise labeling of the data, the real-time monitoring system was combined with high-speed hard X-ray radiography. In particular, the process dynamics have been visualized by X-ray images of the process area. From the X-ray data, the following events are extracted and used for the training of the ML classifier: conduction welding, stable keyhole, unstable keyhole, blowout, and pores.

Compared to the previously described works, the AE signals were sensed by a piezoelectric sensor (PICO HF-1.2, Physical Acoustic, Germany) attached to the sample holder. Moreover, the laser was additionally equipped with an optical system for the collection of the back-reflected light (LBR) from the process zone. Fig. 6 shows a sequence of X-ray images of the process zone during the experiment, together with the recorded $\mathrm{AE}$ and LBR signals. 

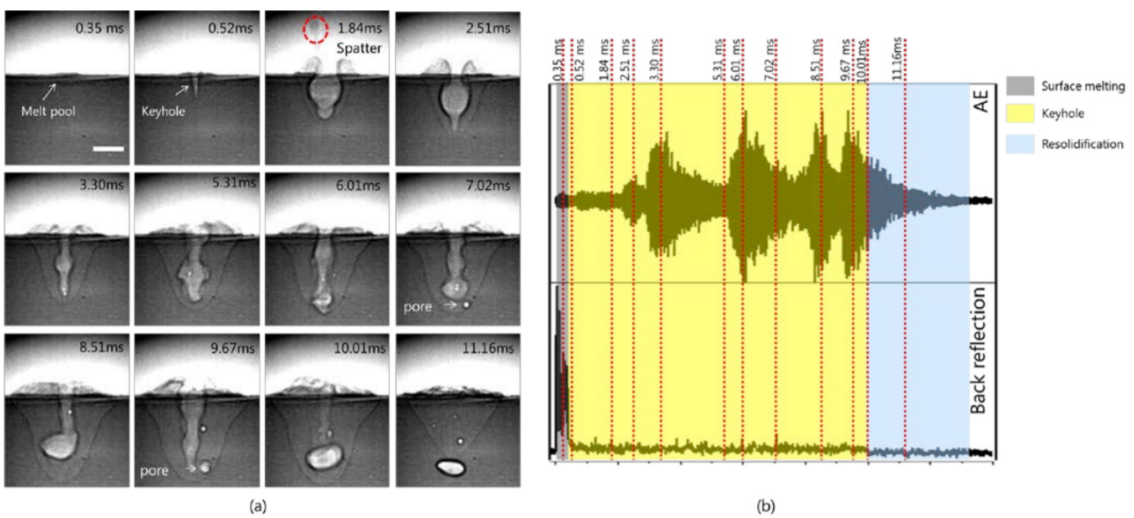

Fig. 6. X-ray images of the process zone (a) and the corresponding AE and optical signals (b) during the experiment at $250 \mathrm{~W}$. The scale bar equals $300 \mu \mathrm{m}$. Reprinted by permission from Elsevier License: Elsevier 39.

The spectrograms computed utilizing the WPT (see Section 2.2.1) of these two signals were then combined, and the corresponding classification accuracy is compared to the results obtained with the individual sensors. As can be seen in Table 2, the unification of LBR and AE improves the accuracy of the classification, although the increment is only marginal compared to what it can be obtained with the AE only.

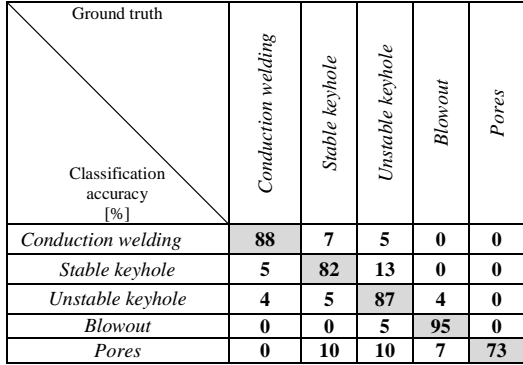

(a)

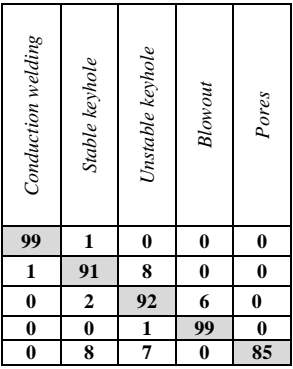

(b)

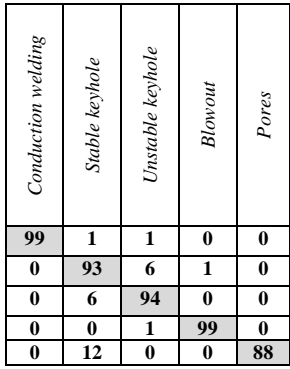

(c)

Table 2. Percentage classification accuracies per category versus ground truth for (a) LBR sensor, (b) AE sensor, and (c) combination of the two sensors. Adapted from Shevchik et al. 26. 


\subsubsection{Results and future work}

There are two remarkable results in this work. First, the X-ray guidance allowed us to provide the ML framework with high quality labeled data, increasing the certainty of the system in distinguishing between stable keyhole and unstable keyhole. Since the latter is subject to defects, the ability to detect the stable unstable transition is of great interest. Indeed, it is a prerequisite for the implementation of a closed-loop process to avoid the formation of defects. Second, it was noticed that it is not only possible to detect the formation of pores, but also their removal through a successive scan of the laser. This outcome is particularly satisfactory since it enables the realization of a device that can first detect defects and successively repair them.

\section{Beyond classification: process control}

As previously mentioned, the developed monitoring system allows the detection of several momentary events responsible for the production of workpieces with different qualities. From the initial offline analysis, we have seen how the system has evolved both in terms of classification (from the generic regimes to the detection of the physical events) and computational speed, making it ready for online applications.

The natural continuation of this work is the development of a system that is not only capable of monitoring the quality of the workpiece but also to predict the upcoming events and to take corrective actions to prevent the onset of defects. Our research group is already working in this direction. In particular, the goal is to apply Machine Learning in this situation as well, in the form of Reinforcement Learning (RL). RL, indeed, is able to learn, via trial and error, an optimal feedback controller for the monitored process.

\section{Conclusions}

The present contribution provides an overview of our research efforts on in situ and real-time monitoring of SLM and LW processes. Our approach primarily relies on the combination of acoustic emission sensing technology and stateof-the-art Machine Learning algorithms. Indeed, even though AE signals can provide information that is not accessible to standard optical methods - such as the volumetric behavior of the process zone - their complicated nature makes it hard for conventional approaches to excerpt meaningful information from them. $\mathrm{ML}$, in contrast, is able to perform a convenient data-driven feature extraction and recognition of the unique acoustic signatures from different quality critic operational regimes.

In particular, our previous works show that the use of ML algorithms for the analysis of the AE signals enables not only the detection of the final qualities of the manufactured pieces but also the momentary events leading to the formation of defects. Furthermore, the system was also able to identify if a subsequent laser pulse has removed a pore, preparing the ground for a defect removal apparatus. 
As of now, the computational time per classification on a PC equipped with a Graphics Processing Unit (GPU) can be as low as $2 \mathrm{~ms}$. This fact, combined with the cost-effectiveness of the AE technology, makes the system ready for the integration in commercially available machines with minimum effort for in situ and real-time monitoring of AM processes.

Quality monitor is only one of the many outcomes of the presented methodology. The described works also open the doors for an AI-based control loop of laser processes. Indeed, based on the gained experience, it is possible to design an adaptive ML control unit the can detect changes in the process dynamics and automatically adjust the process parameters to manufacture high-quality workpieces reproducibly.

In order to achieve this goal, additional investigations are planned for future improvements to the system performance. In fact, despite the promising results based on $\mathrm{AE}$, it is of our belief that the monitoring system can benefit from a combination of different types of sensors, e.g., pyrometer or photodiodes. Specifically, the sensor aggregation can increase the robustness of the system in a noisier environment and enable it to work with a more complicated process. Secondly, an expansion of the quality categories is needed to investigate the applicability of the algorithm to other defect types such as lack of fusion, balling, delamination, and cracking. Finally, the generalization of the monitoring system's capabilities has to be scrutinized by investigating how the algorithm can adapt to new materials or to different geometries.

\section{References}

1. Zhai, Y., Lados, D.A., Lagoy, J.L.: Additive Manufacturing: Making imagination the major Limitation. JOM 66(5), 808-816 (2014)

2. King, W.E., Anderson, A.T., Ferencz, R.M., Hodge, N.E., Kamath, C., Khairallah, S.A., Rubenchik, A.M.: Laser powder bed fusion additive manufacturing of metals; physics, computational, and materials challenges. Applied Physics Reviews 2(4), 041304 (12 2015), http://aip.scitation.org/doi/10.1063/1.4937809

3. Reddy, S.N., Maranan, V., Simpson, T.W., Palmer, T., Dickman, C.J.: Application of topology optimization and design for additive manufacturing guidelines on an automotive component. In: Proceedings of the ASME Design Engineering Technical Conference. vol. 2A-2016. American Society of Mechanical Engineers (ASME) (12 2016)

4. Salmi, M., Tuomi, J., Paloheimo, K.S., Björkstrand, R., Paloheimo, M., Salo, J., Kontio, R., Mesimäki, K., Mäkitie, A.A.: Patient-specific reconstruction with 3D modeling and DMLS additive manufacturing. Rapid Prototyping Journal 18(3), 209-214 (2012)

5. Türk, D., Kussmaul, R., Zogg, M., Klahn, C., Spierings, A.B., Könen, H., Ermanni, P., Meboldt, M.: Additive Manufacturing with Composites for Integrated Aircraft Structures. International SAMPE Technical Conference pp. 1404-1418 (2016)

6. Klotz, U.E., Tiberto, D., Held, F.: Optimization of 18-karat yellow gold alloys for the additive manufacturing of jewelry and watch parts. Gold Bulletin 50(2), 111-121 (6 2017) 
7. Blurhapsody launches 3d printed pasta service (for real this time) / video 3d printing media network, [ONLINE] https://www.3dprintingmedia.network/ blurhapsody-launches-3d-printed-pasta-service-real-time-video/, accessed 2020$02-24$

8. Frazier, W.: Metal additive manufacturing: A review. Journal of Materials Engineering and Performance 23 (06 2014)

9. Das, M., Balla, V.K., Basu, D., Bose, S., Bandyopadhyay, A.: Laser processing of SiC-particle-reinforced coating on titanium. Scripta Materialia 63(4), 438-441 (8 2010)

10. Gu, D.D., Meiners, W., Wissenbach, K., Poprawe, R.: Laser additive manufacturing of metallic components: materials, processes and mechanisms. International Materials Reviews 57(3), 133-164 (5 2012), http://www.tandfonline.com/doi/full/ 10.1179/1743280411Y.0000000014

11. Everton, S.K., Hirsch, M., Stravroulakis, P., Leach, R.K., Clare, A.T.: Review of insitu process monitoring and in-situ metrology for metal additive manufacturing. Materials Design 95, 431 - 445 (2016), http://www.sciencedirect.com/science/ article/pii/S0264127516300995

12. Tapia, G., Elwany, A.: A review on process monitoring and control in metal-based additive manufacturing. Journal of Manufacturing Science and Engineering 136, 060801 (12 2014)

13. Tammas-Williams, S., Zhao, H., Léonard, F., Derguti, F., Todd, I., Prangnell, P.B.: XCT analysis of the influence of melt strategies on defect population in Ti$6 \mathrm{Al}-4 \mathrm{~V}$ components manufactured by Selective Electron Beam Melting. Materials Characterization 102, 47-61 (4 2015)

14. Shifeng, W., Shuai, L., Qingsong, W., Yan, C., Sheng, Z., Yusheng, S.: Effect of molten pool boundaries on the mechanical properties of selective laser melting parts. Journal of Materials Processing Technology 214(11), 2660-2667 (2014)

15. Lewandowski, J.J., Seifi, M.: Metal Additive Manufacturing: A Review of Mechanical Properties. Annual Review of Materials Research 46(1), 151-186 (7 2016), http://www.annualreviews.org/doi/10.1146/annurev-matsci-070115-032024

16. Thompson, A., Maskery, I., Leach, R.: X-ray computed tomography for additive manufacturing: A review. Measurement Science and Technology 27, 072001 (06 2016)

17. Sharratt, B.M.: Non-Destructive Techniques and Technologies for Qualification of Additive Manufactured Parts and Processes: A Literature Review. Tech. rep. (2015)

18. Scime, L., Beuth, J.: Anomaly detection and classification in a laser powder bed additive manufacturing process using a trained computer vision algorithm. Additive Manufacturing 19, 114-126 (1 2018)

19. Martin, A.A., Calta, N.P., Khairallah, S.A., Wang, J., Depond, P.J., Fong, A.Y., Thampy, V., Guss, G.M., Kiss, A.M., Stone, K.H., Tassone, C.J., Nelson Weker, J., Toney, M.F., van Buuren, T., Matthews, M.J.: Dynamics of pore formation during laser powder bed fusion additive manufacturing. Nature Communications 10(1), 1-10 (12 2019)

20. Krampikowska, A., Pała, R., Dzioba, I., Šwit, G.: The use of the acoustic emission method to identify crack growth in 40CrMo steel. Materials 12(13) (7 2019)

21. Mazal, P., Vlasic, F., Koula, V.: Use of Acoustic Emission Method for Identification of Fatigue Micro-cracks Creation. In: Procedia Engineering. vol. 133, pp. 379-388. Elsevier Ltd (1 2015)

22. Saeidi, F., Shevchik, S.A., Wasmer, K.: Automatic detection of scuffing using acoustic emission. Tribology International 94, 112-117 (2 2016) 
23. Shevchik, S.A., Meylan, B., Mosaddeghi, A., Wasmer, K.: Acoustic Emission for in Situ Monitoring of Solid Materials Pre-Weakening by Electric Discharge: A Machine Learning Approach. IEEE Access 6, 40313-40324 (7 2018)

24. Shevchik, S.A., Kenel, C., Leinenbach, C., Wasmer, K.: Acoustic emission for in situ quality monitoring in additive manufacturing using spectral convolutional neural networks. Additive Manufacturing 21, 598-604 (5 2018)

25. Shevchik, S.A., Le-Quang, T., Farahani, F.V., Faivre, N., Meylan, B., Zanoli, S., Wasmer, K.: Laser welding quality monitoring via graph support vector machine with data adaptive kernel. IEEE Access 7, 93108-93122 (2019)

26. Shevchik, S., Le-Quang, T., Meylan, B., Farahani, F.V., Olbinado, M.P., Rack, A., Masinelli, G., Leinenbach, C., Wasmer, K.: Supervised deep learning for real-time quality monitoring of laser welding with X-ray radiographic guidance. Scientific Reports 2020 10:1 10(1), 1-12 (2 2020)

27. Mallat, S., Hwang, W.L.: Singularity detection and processing with wavelets. IEEE Transactions on Information Theory 38(2), 617-643 (1992)

28. Daubechies, I.: Ten Lectures on Wavelets. Society for Industrial and Applied Mathematics (1 1992)

29. Jolliffe, I., Cadima, J.: Principal component analysis: A review and recent developments. Philosophical Transactions of the Royal Society A: Mathematical, Physical and Engineering Sciences 374, 20150202 (04 2016)

30. Goodfellow, I., Bengio, Y., Courville, A.: Deep Learning. MIT Press (2016), http: //www.deeplearningbook.org

31. Shevchik, S.A., Masinelli, G., Kenel, C., Leinenbach, C., Wasmer, K.: Deep learning for in situ and real-time quality monitoring in additive manufacturing using acoustic emission. IEEE Transactions on Industrial Informatics 15(9), 5194-5203 (2019)

32. Piveta, A.C.G., Montandon, A.A.B., Ricci, W.A., Nagle, M.M.: Mechanical strength and analysis of fracture of titanium joining submitted to laser and tig welding. Materials Research 15(6), 937-943 (11 2012)

33. Svenungsson, J., Choquet, I., Kaplan, A.F.: Laser Welding Process - A Review of Keyhole Welding Modelling. In: Physics Procedia. vol. 78, pp. 182-191. Elsevier B.V. (1 2015)

34. Zhou, J., Tsai, H.L., Wang, P.C.: Transfort phenomena and keyhole dynamics during pulsed laser welding. Journal of Heat Transfer 128(7), 680-690 (7 2006)

35. Berger, P., Hügel, H.: Fluid dynamic effects in keyhole welding an attempt to characterize different regimes. In: Physics Procedia. vol. 41, pp. 216-224. Elsevier B.V. (1 2013)

36. Belkin, M., Niyogi, P., Sindhwani, V.: Manifold regularization: A geometric framework for learning from labeled and unlabeled examples. Journal of Machine Learning Research 7, 2399-2434 (11 2006)

37. Melacci, S., Belkin, M.: Laplacian support vector machines trained in the primal. J. Mach. Learn. Res. 12(null), 1149-1184 (Jul 2011)

38. Courtois, M., Carin, M., Le Masson, P., Gaied, S., Balabane, M.: A complete model of keyhole and melt pool dynamics to analyze instabilities and collapse during laser welding. Journal of Laser Applications 26(4), 042001 (11 2014), http: //lia.scitation.org/doi/10.2351/1.4886835

39. Le Quang, T., Shevchik, S., Meylan, B., Vakili-Farahani, F., Olbinado, M., Rack, A., Wasmer, K.: Why is in situ quality control of laser keyhole welding a real challenge? 74, 649-653 (09 2018) 